\title{
Loss Mechanisms for T-ray Microwires
}

\author{
Shaghik Atakaramians ${ }^{1,2}$, Shahraam Afshar Vahid ${ }^{2}$, Bernd M. Fischer ${ }^{1}$, Derek Abbott ${ }^{1}$ and Tanya M. Monro ${ }^{2}$ \\ ${ }^{1}$ Centre for Biomedical Engineering and School of Electrical \& Electronic Engineering, \\ ${ }^{2}$ Centre of Expertise in Photonics and School of Chemistry \& Physics, \\ The University of Adelaide, SA 5005 Australia \\ shaghik@eleceng.adelaide.edu.au
}

\begin{abstract}
In this paper we will present predictions for loss mechanisms caused by material, waveguide, surface roughness and bends in microwires and estimate their affect on the total loss in the terahertz regime.
\end{abstract}

\section{INTRODUCTION}

The terahertz $(\mathrm{THz})$ or T-ray region of electromagnetic spectrum, located between millimeter wave and infrared frequencies, has attracted much interest over the last decade. Terahertz spectroscopic techniques have many applications as for example in detection of many biological and chemical materials [1]. In almost all existing terahertz time domain spectroscopy systems terahertz waves propagate in free space and at present low loss terahertz waveguides are not available.

Several waveguide solutions based on technologies from both electronics and photonics have been studied such as the hollow metallic circular waveguide [2], [3], hollow metallic rectangular waveguide [3], sapphire fiber [4], plastic ribbon waveguide [5], air-filled parallel-plate waveguide [6], [7], plastic photonic crystal fiber [8], coaxial waveguide [9], metal wire waveguide [10], [11], parallel-plate photonic waveguide [12], metal sheet waveguide [13], the dielectric-filled parallel-plate waveguide [14], low-index discontinuity terahertz waveguides (split rectangular and tube waveguides) [15], and metallic slit waveguide [16].

Metallic slit waveguides and bare metal wires are promising guiding techniques reported in the literature, and attenuation constants less than $0.07 \mathrm{~cm}^{-1}$ and $0.03 \mathrm{~cm}^{-1}$, respectively have been reported [16] and [10]. Chen et al. [17] have recently reported loss values less than $0.01 \mathrm{~cm}^{-1}$ near 0.3 $\mathrm{THz}$ in plastic fibers. The concept underlying the waveguiding properties of $\mathrm{THz}$ radiation in these fibers is analogous to optical nanowire fibers [18], since in these fibers, a significant fraction of the guided light travels outside the material. For this reason we have coined the term microwires for these waveguides used in the terahertz regime.

In general, there are different loss mechanisms that should be considered when we are dealing with fibers with subwavelength core dimensions. Such loss mechanisms are: material and waveguide losses, and scattering loss due to surface roughness and bend loss, which can couple light from bound modes to the radiation modes.

\section{LOSS MECHANISMS}

\section{A. Material and Waveguide Losses}

Based on optical measurements of the bulk material loss shown in Fig. 1 [19], and solving the vectorial form of Maxwell's equation for microwire structures, we have calculated the effective material and waveguide loss, which is defined as the average of the loss coefficients inside (dielectric) and outside (air) the fiber over the transverse field distributions [19]. Figure 2(a) shows the results of effective loss for a polymer, a range of soft glasses, and a diamond. In the microwire regime, where the fiber diameter is much lower than the operating wavelength the effective loss approaches to a value similar for all materials, as shown in Fig. 2(b).

\section{B. Bend loss}

Radiative losses occur whenever the fiber undergoes a bend of finite radius curvature. If the radius of the bend is larger than the fiber diameter, it is known as macrobending loss, or simply bending loss. As the radius of curvature decreases the loss increases exponentially until at a certain critical radius, the bend loss of the fundamental mode is equal to $3 \mathrm{~dB}$ per loop. For bends tighter than the critical radius, the bend loss increases dramatically and little light is transmitted [20].

Figure 3(a) shows the bend loss for a PMMA microwire with three bend radii $(100 \mathrm{~m}, 60 \mathrm{~m}$ and $40 \mathrm{~m})$ at $f=0.5$ $\mathrm{THz}(\lambda=600 \mu \mathrm{m})$ versus fiber diameter. Bend loss increases by decreasing the bend radius, therefore in order to benefit from low loss propagation in microwires, the structure should be kept straight. As long as the bend radius is larger than 100

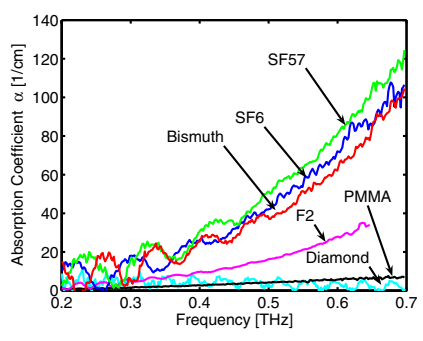

(a)

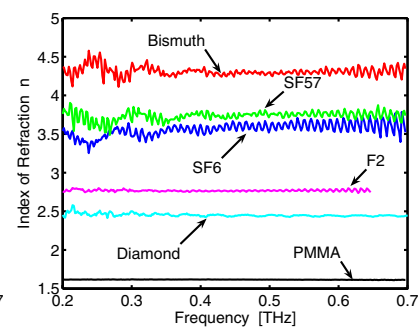

(b)
Fig. 1. (a) Absorption coefficients and (b) the refractive indices of the bulk materials (Diamond, PMMA, F2, SF6, SF57 and Bismuth glass) measured with a $\mathrm{THz}$ time domain spectrometer. 


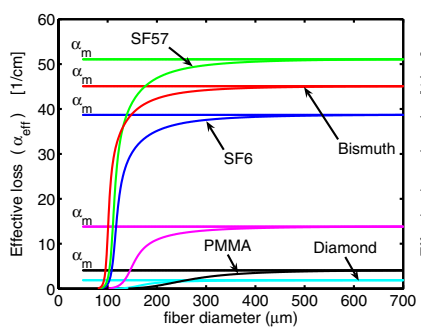

(a)

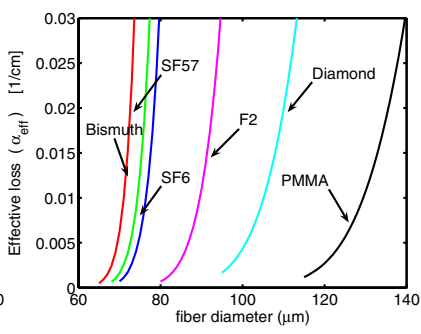

(b)
Fig. 2. (a) Effective loss of fibers made up of Diamond, PMMA, F2, SF6, SF57 and Bismuth glass materials versus fiber diameter at $f=0.5 \mathrm{THz}$ $(\lambda=600 \mu \mathrm{m})$. (b) Magnification of the lower limit of the effective loss shown in Figure 2(a)

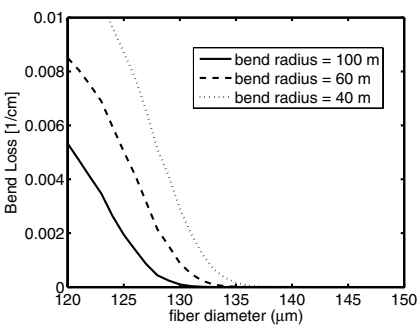

(a)

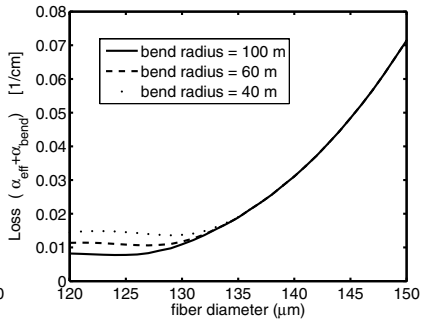

(b)
Fig. 3. (a) Bend loss of a PMMA microwire versus fiber diameter, for three bend radii at $f=0.5 \mathrm{THz}(\lambda=600 \mu \mathrm{m})$ (b) Effective and bend losses of a PMMA fiber with 40, 60 and 100 meters bend radii.

$\mathrm{m}$, a very gentle bend, the total loss of the structure will still be less than $0.01 \mathrm{~cm}^{-1}$. This is depicted in Figure $3(\mathrm{~b})$ for a PMMA microwire. An increase in bend radius leads to a shift in value and position of the minimum achievable total loss, which is the same of the effective loss and bend loss. It can be shown that the behavior occurs for the other microwire materials.

\section{DisCUSSION AND CONCLUSION}

In addition to the loss mechanisms mentioned above, the presence of a glass-air interface in fibers introduces new mechanisms of scattering loss, which is due to the inherent roughness of the interface [21], [22]. This is a limiting factor in achieving a minimum loss specially for optical photonic crystal fibers [22]. However, for terahertz microwires, it is expected that the surface roughness loss would be substantially less than the other losses for microwires due to the fact that the operating wavelength is larger than the height of the surface roughness perturbation. This is consistent with previous reports that surface roughness loss decreases as a function of $\lambda[22]$.

In conclusion, the dominant loss mechanisms for the microwires are effective material and waveguide losses, and bend loss. The smaller the diameter of the microwire, the lower the effective loss, which results in less confinement of the field in the structure and therefore increasing the loss due to bends.
Rigid and straight microwires are promising solutions for low loss terahertz transmission.

\section{ACKNOWLEDGMENT}

We gratefully acknowledge David N. Jamieson for supplying the diamond sample, and Naoki Sugimoto of Asahi Glass Japan for supplying the bismuth glass sample.

\section{REFERENCES}

[1] K. Sakai, Terahertz, Optoelectronics. Berlin, Heidelberg: Springer, 2005

[2] R. McGowan, G. Gallot, and D. Grischkowsky, "Propagation of ultrawideband short pulses of terahertz radiation through submillimeterdiameter circular waveguides," Optics Letters, vol. 24, pp. 1431-1433, 1999.

[3] G. Gallot, S. Jamison, R. McGowan, and D. Grischkowsky, "Terahertz waveguides," Journal of the Optical Society of America B, vol. 17, pp. 851-863, 2000

[4] S. Jamison, R. McGowan, and D. Grischkowsky, "Singlemode waveguide propagation and reshaping of sub-ps terahertz pulses in sapphire fibers," Applied Physics Letters, vol. 76, pp. 1987-1989, 2000.

[5] R. Mendis and D. Grischkowsky, "Plastic ribbon $\mathrm{THz}$ waveguides," Journal of Applied Physics, vol. 88, pp. 4449-4451, 2000.

[6] _ - "Undistorted guided-wave propagation of subpicosecond terahertz pulses," Optics Letters, vol. 26, pp. 846-848, 2001.

[7] - "THz interconnect with low-loss and low-group velocity dispersion," IEEE Microwave and Wireless Components Letters, vol. 26, pp. 444-446, 2001.

[8] H. Han, H. Park, M. Cho, and J. Kim, "Terahertz pulse propagation in a plastic photonic crystal fiber," Applied Physics Letters, vol. 80, pp. 2634-2636, 2002.

[9] T.-I. Jeon and D.Grischkowsky, "Direct optoelectronic generation and detection of sub-ps-electrical pulses on sub-mm-coaxial transmission lines," Applied Physics Letters, vol. 85, pp. 6092-6094, 2004.

[10] K. Wang and D. M. Mittleman, "Metal wires for terahertz wave guiding," Nature, vol. 432, pp. 376-379, 2004.

[11] T.-I. Jeon, J. Zhang, and D. Grischkowsky, "THz Sommerfeld wave propagation on a single metal wire," Applied Physics Letters, vol. 86, 161904,2005

[12] A. Bingham, Y. Zhao, and D. Grischkowsky, "THz parallel plate photonic waveguide," Applied Physics Letters, vol. 87, 051101, 2005.

[13] T.-I. Jeon and D. Grischkowsky, "THz Zenneck surface wave (THz surface plasmon) propagation on a metal sheet," Applied Physics Letters, vol. 88, 061113, 2006.

[14] R. Mendis, "Nature of subpicosecond terahertz pulse propagation in practical dielectric-filled parallel-plate waveguides," Optics Letters, vol. 31 , no. 17 , pp. 2643-2645, 2006.

[15] M. Nagel, A. Marchewka, and H. Kurz, "Low-index discontinuity terahetz waveguides," Optics Express, vol. 14, no. 21, pp. 9944-9954, 2006.

[16] M. Wachter, M. Nagel, and H. Kurz, "Metallic slit waveguide for dispersion-free low-loss terahertz signal transmission," Applied Physics Letters, vol. 90, 061111,2007.

[17] L.-J. Chen, H.-W. Chen, T.-F. Kao, J.-Y. Lu, and C.-K. Sun, "Low-loss subwavelength plastic fiber for terahertz waveguiding," Optics Letters, vol. 83, no. 18, pp. 3656-3658, 2006.

[18] L. Tong, R. R. Gattass, J. B. Ashcom, S. He, J. Lou, M. Shen, I. Maxwell, and E. Mazur, "Subwavelength-diameter silica wires for low-loss optical wave guiding," Nature, vol. 426, pp. 3656-3658, 2003.

[19] S. Atakaramians, S. Afshar Vahid, B. Fischer, H. Ebendorff-Heidepriem, T. Monro, and D. Abbott, "Low loss terahertz transmission," in Proceedings SPIE Micro- and Nanotechnology: Smart Materials, Nano- and Micro-Smart Systems, vol. 6414, art. no. 64140I, Adelaide, Australia, 10-13 Dec., 2006.

[20] A. W. Snyder and J. D. Love, Optical Waveguide Theory. London: Chapman and Hall, 1995.

[21] J. Jäckle and K. Kawasaki, "Intrinsic roughness of glass surfaces," $J$. Phys.: Condens. Matter, vol. 7, pp. 4351-4358, 1995.

[22] P. Roberts, F. Couny, H. Sabert, B. Mangan, T. Birks, J. Knight, and P. Russell, "Loss in solid-core photonic crystal fibers due to interface roughness scattering," Optics Express, vol. 13, no. 20, pp. 7779-7793, 2005 . 